\title{
Évolution des peuplements de crustacés du Rhône Moyen au cours des 20 dernières années, relation avec la variabilité hydroclimatique
}

\section{Long-term changes in the Crustaceans fauna of the Middle Rhône River consequently to hydroclimatic events}

\author{
J. Dessaix, J.-F. Fruget \\ ARALEP, Écologie des Eaux Douces, Domaine Scientifique de la Doua, Bât. CEl, 66 Bld Niels Bohr, \\ BP 2132, 69603 Villeurbanne Cedex, France \\ e-mail : dessaix@aralep.com - fruget@aralep.com
}

\begin{abstract}
Résumé - Le suivi hydrobiologique du CNPE de Saint-Alban situé sur le Rhône Moyen montre une diminution régulière et marquée des populations de crustacés autochtones depuis une dizaine d'années, en particulier des Aselles. Parallèlement à cela, plusieurs espèces de crustacés invasifs sont progressivement apparues. Une étude spécifique des populations de crustacés et de leurs dynamiques temporelles a donc été réalisée sur un cycle annuel de février 2006 à janvier 2007. L'étude de la chronique de température depuis le début du suivi du site au milieu des années 1980 met en évidence une dérive des températures sur la période considérée, s'accentuant sur les dernières années de la chronique, parallèlement à d'autres événements ponctuels importants tels que les épisodes de crue et de canicule. La station aval réchauffée montre une précocité des différents seuils thermiques et un raccourcissement de la période froide. Du point de vue biologique, une première vague de crustacés invasifs (G. tigrinus, $D$. villosus) a été notée durant les années 1990 sur le Rhône Moyen, principalement en relation avec d'importants épisodes hydrologiques qui accélèrent ces échanges initiés à l'origine par le développement des liaisons fluviales inter-bassins. La période de canicule et post canicule du début des années 2000 se traduit ensuite par le développement d'une seconde vague d'espèces exotiques invasives ( $A$. desmarestii, $H$. anomala, $C$. curvispinum) et l'installation durable des premiers arrivants. Le suivi spécifique mensuel durant un an des populations de crustacés a permis de préciser diverses pistes de réflexion: (i) augmentation des effectifs hivernaux pour certaines espèces (C. curvispinum et G. tigrinus en particulier) ; (ii) limitation de certaines populations à la station aval réchauffée (A. aquaticus, G. pulex, G. fossarum, A. desmarestii); (iii) modification du cycle biologique (reproduction plus précoce mais de plus courte durée de $D$. villosus dans le secteur aval); (iv) confirmation d'éventuels phénomènes de compétition interspécifique (G. tigrinus et $D$. villosus). Ainsi, afin d'apprécier les modifications des interactions biotiques engendrées par les stress environnementaux que sont la variabilité hydroclimatique et les invasions biologiques, des études plus poussées faisant appel à la biologie des populations et à l'écologie évolutive s'avèrent nécessaires (potentiel adaptatif des individus à la thermie en particulier).
\end{abstract}

Article published by EDF and available at $h$ ttp://www.hydroecologie.org

or http://dx.doi.org/10.1051/hydro/2009001 
Mots clés - crustacés, Rhône, température, invasions biologiques, suivi long-terme

\begin{abstract}
The hydrobiological monitoring of Saint-Alban nuclear power plant located on the Middle Rhône River shows a regular and marked decrease of the populations of native Crustaceans since about ten years, in particular of Asellids. Concurrently, several invasive Crustaceans species gradually appeared. A specific study of the Crustaceans populations and of their temporal dynamics was thus carried out on an annual cycle from February 2006 to January 2007. The study of the temperature time series since the beginning of the survey of the site during the middle of the 1980s highlights a drift of the temperatures over the period considered, being accentuated over the last years of the chronicle, parallel to other important specific events such as the episodes of flood and heat wave. The heated downstream station shows precocity of the various thermal thresholds and a shortening of the cold period. From the biological point of view, a first wave of alien Crustaceans (G. tigrinus, D. villosus) was noted during the 1990s on the Middle Rhône River, mainly in relation to important hydrological events which accelerate these exchanges initiated originally by the development of the between-basins waterways. The period of heat wave and post heat wave of the beginning of the 2000s results then in the development of a second wave of invasive exotic species (A. desmarestii, $H$. anomala, $C$. curvispinum) and the durable installation of the first invaders. The monthly specific survey during one year of the Crustaceans populations made it possible to specify various tracks of reflexion: (i) increase in winter strength for certain species (C. curvispinum and G. tigrinus in particular); (ii) limitation of certain populations at the heated downstream station (A. aquaticus, $G$. pulex, G. fossarum, A. desmarestii); (iii) modification of the biological cycle (earlier reproduction but of shorter duration of $D$. villosus in the downstream sector); (iv) confirmation of possible phenomena of interspecific competition (G. tigrinus and $D$. villosus). Thus, in order to appreciate the modifications of the biotic interactions generated by the environmental stresses that are the hydroclimatic variability and the biological invasions, more particular studies such as populations biology and evolutionary ecology (adaptive potential of the individuals to temperature increase in particular) are needed.
\end{abstract}

Key words - crustaceans, Rhône River, temperature, biological invasions, long-term series

\section{INTRODUCTION}

Le suivi hydrobiologique du CNPE de Saint-Alban situé sur le Rhône Moyen à une cinquantaine de kilomètres en aval de Lyon (Fruget et al., 1999) montre une diminution régulière et marquée des populations de crustacés depuis une dizaine d'années, en particulier des Aselles. Cette baisse est particulièrement sensible à la station située en aval du site où Asellus aquaticus, autrefois parmi les taxons dominants, devient de plus en plus « anecdotique ». Parallèlement à cela, plusieurs espèces de crustacés invasifs sont progressivement apparues (Dikerogammarus villosus, Gammarus tigrinus, Crangonyx pseudogracilis, Chelicorophium curvispinum, Atyaephyra desmarestii, entre autres), accroissant rapidement leurs effectifs pour certains d'entre eux (Dikerogammarus).

La baisse des effectifs d'Aselles a débuté antérieurement à l'extension de Dikerogammarus et s'est amplifiée depuis. II est par ailleurs avéré que le mélange incomplet dans un premier temps de l'eau réchauffée et de l'eau 
du fleuve induit l'existence de zones hétérogènes de température en aval des CNPE, à l'origine potentielle de différences biologiques locales, principalement au niveau des zones rivulaires (Bady \& Fruget, 2006). Quelle est la part de ce phénomène thermique avec sa chronicité et la permanence de l'échauffement de la zone rivulaire (environ $+5^{\circ} \mathrm{C}$ par rapport à l'amont) dans les modifications enregistrées parmi les populations de crustacés? Qu'en est-il du phénomène de compétition avec Dikerogammarus? Quel est le comportement du nouvel arrivant par rapport au facteur thermique?

Le suivi annuel ne permet pas d'appréhender finement ces différentes interrogations, son protocole n'étant pas fait pour cela. Une étude spécifique des populations de crustacés et de leurs dynamiques temporelles a donc été réalisée sur un cycle annuel, en marge du suivi scientifique de routine.

\section{MATÉRIEL ET MÉTHODES}

\subsection{Stations d'étude}

Trois stations du suivi réglementaire sont plus particulièrement concernées par cette étude spécifique : d'une part la station de référence en amont du site (pk 47) et la station aval proche (pk 49), toutes deux situées sur la retenue de l'aménagement CNR de Péage-de-Roussillon, d'autre part la partie du Rhône court-circuitée ou RCC (pk 54,5), référence plus « naturelle ». Le canal de rejet de l'eau réchauffée débouche dans le fleuve aux environs du pk 48,5 sur la retenue (Fig. 1 et Photo 1 ).

\subsection{Méthodologie d'étude}

L'échantillonnage est réalisé par substrats artificiels de type IBGA mis au point dans le cadre du suivi de Saint-Alban (type mixte ficelle-cailloux - Photo 2). Ces substrats sont déposés près des rives, zones où s'effectue préférentiellement l'échauffement à la station aval.

Un relevé mensuel des substrats est effectué durant un an toutes les 4 semaines, intervalle qui représente le temps de colonisation et de stabilisation nécessaire (Khalaf \& Tachet, 1978), soit un total de 12 mois d'échantillonnage, permettant de couvrir un cycle biologique. Le premier relevé a été effectué le 27 février 2006. Le second échantillon de la partie court-circuitée a été perdu durant la crue de mars-avril.

Tous les crustacés, tant Gammaridés qu'Asellidés ou autres, sont récoltés, déterminés à l'espèce et comptés, mais l'étude de dynamique des populations est plus spécifiquement axée sur Asellus aquaticus et Dikerogammarus villosus.

Les paramètres suivant sont considérés : effectifs des différentes espèces, proportion mâles/femelles chez les adultes, état de maturité des femelles (ovigères ou non), proportion d'individus juvéniles, différenciation de classes de taille.

Parallèlement à ces relevés biologiques, des enregistreurs thermiques ont été posés durant la durée de l'étude aux mêmes endroits d'implantation que les substrats afin d'avoir précisément l'évolution de la température locale et de contrôler la synchronicité ou non des seuils thermiques saisonniers entre les différentes stations. 


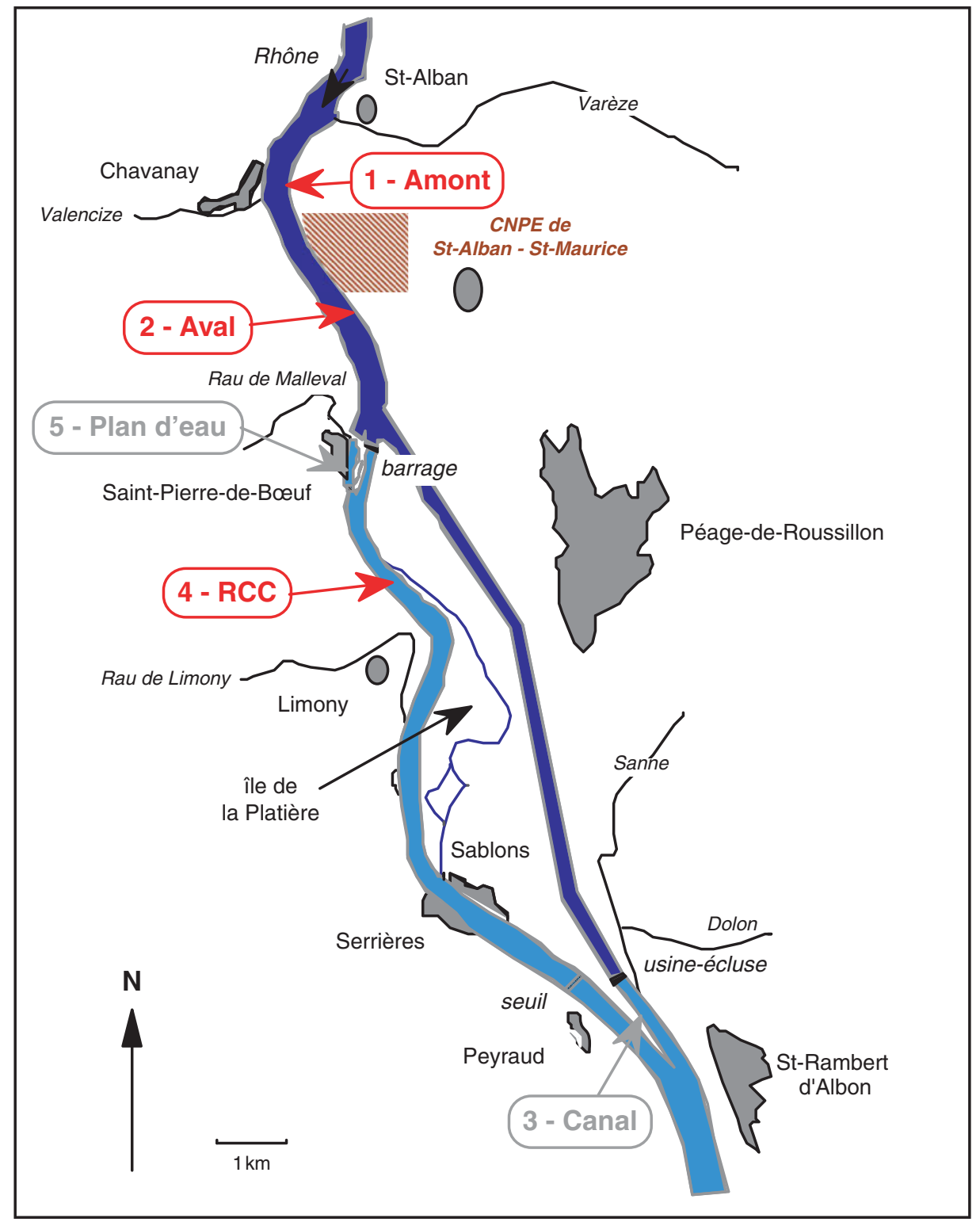

Fig. 1. Localisation des stations d'échantillonnage amont, aval proche et RCC au sein de l'aménagement CNR de Péage-de-Roussillon.

Fig. 1. Location of the sampling sites (upstream, downstream close and by-passed section) within Péage-de-Roussillon scheme. 


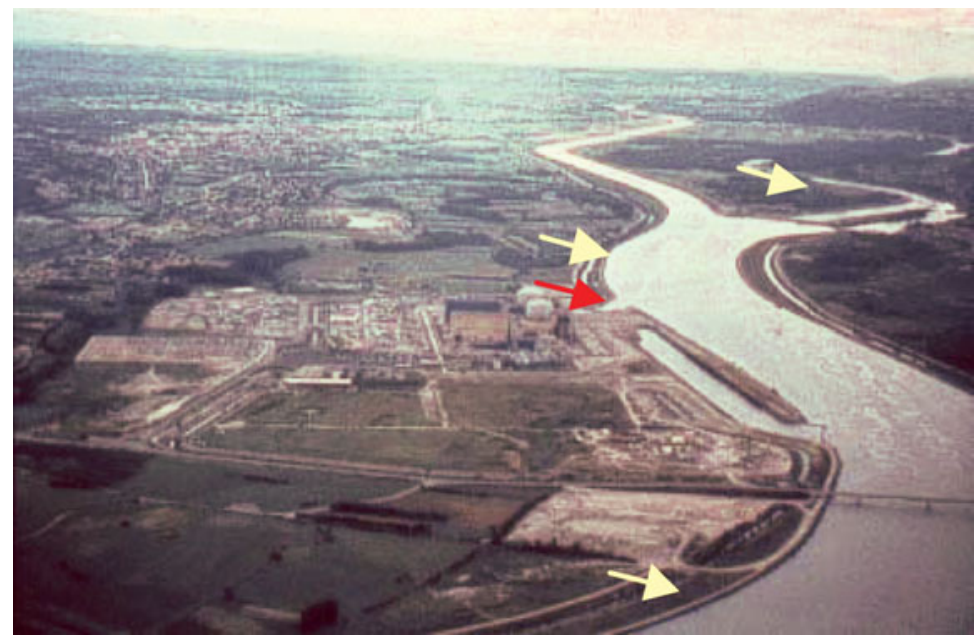

Photo 1. Vue aérienne de l'aménagement de Péage-de-Roussillon et du site de Saint-Alban (Cliché : Frapna 26). Les stations d'étude sont localisées par les flèches jaunes et le rejet du CNPE par la flèche rouge.

Photo 1. Aerial view of Péage-de-Roussillon scheme and the site of Saint-Alban (Picture: Frapna 26). The study sites are located by the yellow arrows and the output of the power plant by the red arrow.

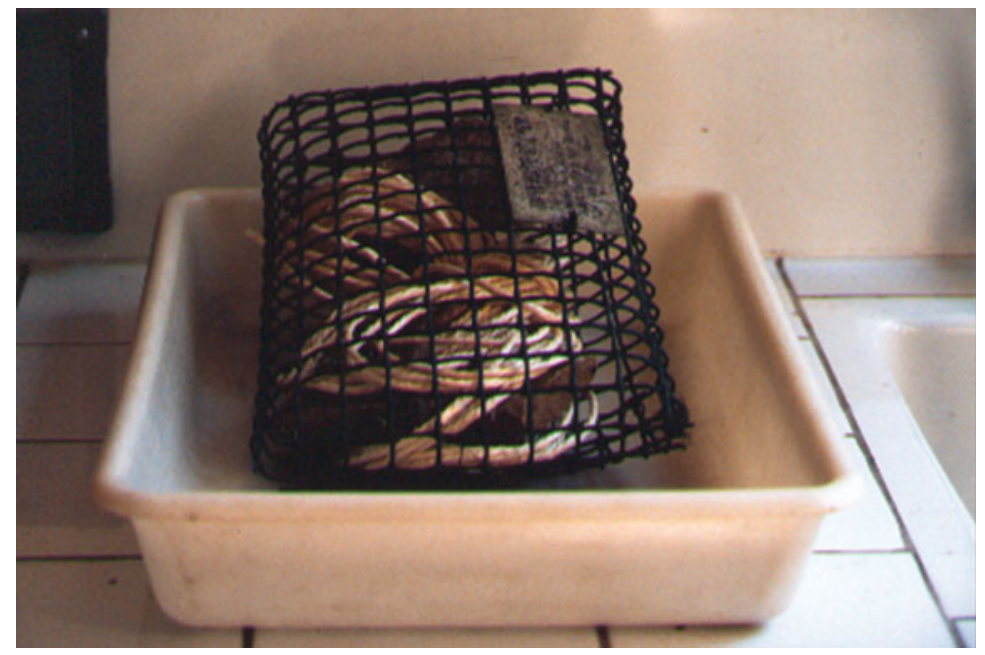

Photo 2. Substrat artificiel mixte type IBGA (Cliché : J.F. Fruget).

Photo 2. Artificial substrate IBGA type (Picture: J.F. Fruget). 


\section{HYDROLOGIE ET TEMPÉRATURE}

\section{1 Évolution inter-annuelle}

La figure 2 présente l'historique de l'hydrologie et de la thermie depuis le début du suivi du site de SaintAlban en 1985. S'il apparaît difficile de dégager des tendances évolutives significatives à cette échelle d'observation, les moyennes mobiles des cinq dernières années font toutefois apparaître une baisse de l'hydrologie générale et une hausse de la température moyenne annuelle. De la même manière, le rapport de synthèse 19852001 du CNPE de Saint-Alban a montré une tendance nette à une baisse de l'hydrologie et à un accroissement progressif de la température lors de la période estivale au cours des dernières années (ARALEP, 2003). Ceci a été confirmé à l'échelle globale du fleuve, avec en particulier des seuils thermiques de plus en plus précoces et une diminution de durée de la période froide en raison d'une prolongation automnale des températures élevées (Carrel, 2006).

\section{Température}

La moyenne des températures moyennes annuelles de la période 1988-2006 est de $14,1^{\circ} \mathrm{C}(\mathrm{N}=19-$ écart type $=0,56$ ). L'écart entre la température moyenne de l'année la plus froide $\left(1993-12,8^{\circ} \mathrm{C}\right)$ et celle de l'année la plus chaude (2003 $15,2^{\circ} \mathrm{C}$ ) est sensible. La comparaison des températures annuelles du Rhône en amont du site fait ressortir une succession d'années " chaudes » et d'années plus "froides », avec une tendance à l'accroissement progressif à partir du milieu des années 1990, s'accentuant sur les dernières années de la chronique (Fig. 2A). La moyenne mobile sur 5 années passe ainsi de $13,6^{\circ} \mathrm{C}$ en 1995 à $13,9^{\circ} \mathrm{C}$ en 2000 et $14,4^{\circ} \mathrm{C}$ en 2006.

Les différentes valeurs enregistrées lors de l'année 2003 sont les plus élevées de la chronique de suivi, tant en moyennes annuelles (environ $15,0^{\circ} \mathrm{C}$ à l'amont, à égalité avec 1989 , et $16,4^{\circ} \mathrm{C}$ pour le RCC), qu'en moyennes mensuelles (respectivement 24,9 et $25,4^{\circ} \mathrm{C}$ pour le mois d'août à l'amont et dans le RCC) ou en valeurs instantanées (respectivement 27,1 et $29,0^{\circ} \mathrm{C}$ en août à l'amont et dans le RCC).

\section{Hydrologie}

Les figures $2 \mathrm{~B}$ et $2 \mathrm{C}$ font ressortir une succession de périodes hydrologiquement déficitaires (19891991, 1996-1997, 2003-2004) et de périodes excédentaires (1987-1988, 1994-1995, 1999-2002) sur le Rhône, avec des crues particulièrement notables en octobre 1993, janvier 1994, février 1995, mars 2001 et novembre 2002 (coefficients mensuels d'hydraulicité $>2$, et jusqu'à 3,5 en octobre 1993).

\section{2 Évolution en 2006}

\section{Hydrologie}

La figure 3 donne un aperçu de l'évolution du débit durant l'étude. Si l'hydraulicité est largement excédentaire aux mois de mars et avril, elle est devenue progressivement déficitaire, en particulier en juin et juillet, mois ayant subi un début d'épisode 

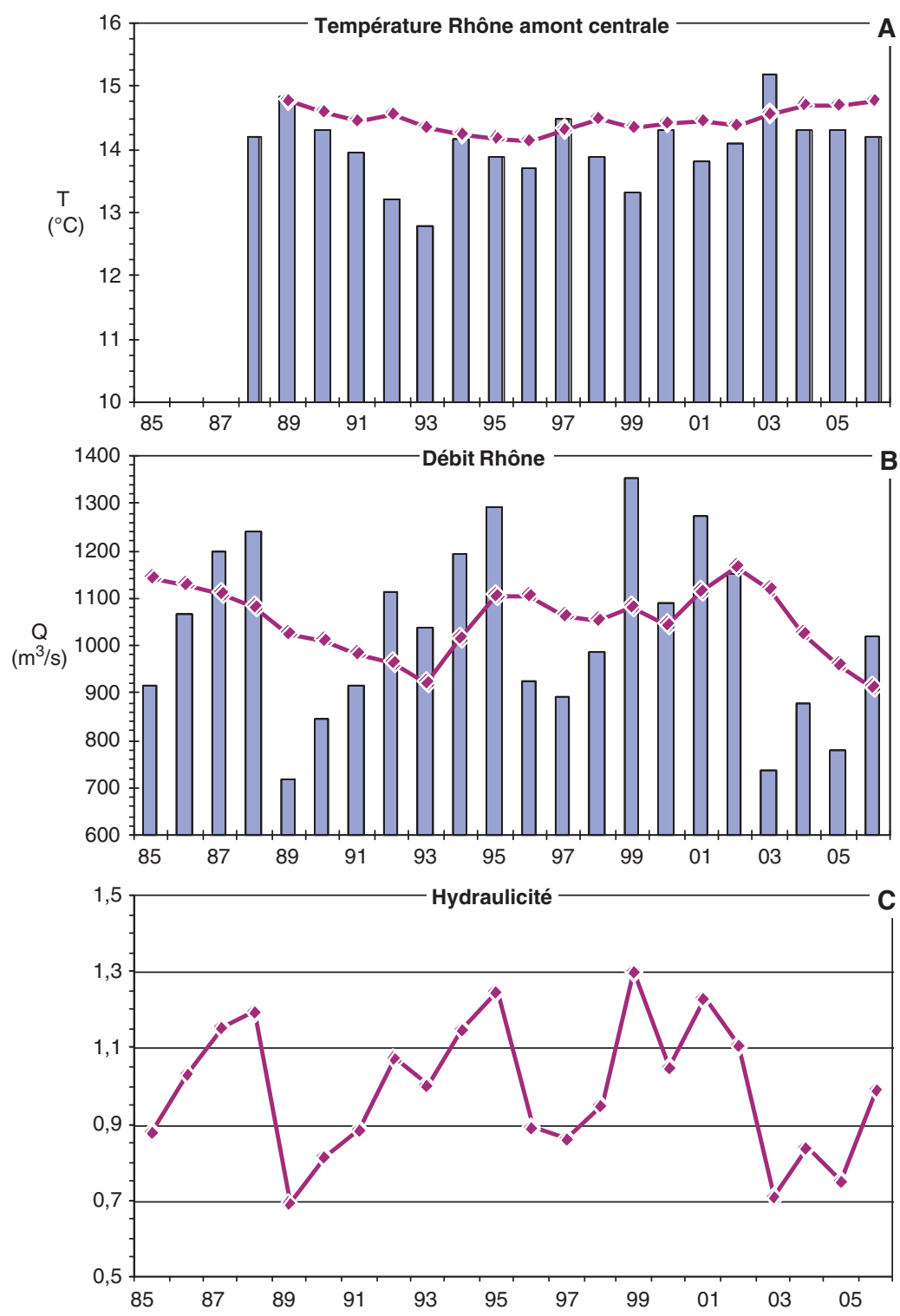

Fig. 2. Évolution inter-annuelle (A) de la température moyenne annuelle du Rhône en amont du site (histogrammes) et de la moyenne mobile correspondante sur 5 années (diamants); (B) du débit moyen annuel du Rhône et de la moyenne mobile sur 5 ans; (C) du coefficient d'hydraulicité. Données CNR (débit) et EDF Saint-Alban (température).

Fig. 2. Between years evolution (A) of the annual average temperature of the Rhône upstream of the site (histograms) and the corresponding moving average over 5 years (diamonds); (B) of the annual medium discharge of the Rhône and the moving average over 5 years; (C) of the coefficient of hydraulicity. Data CNR (discharge) and EDF Saint-Alban (temperature). 


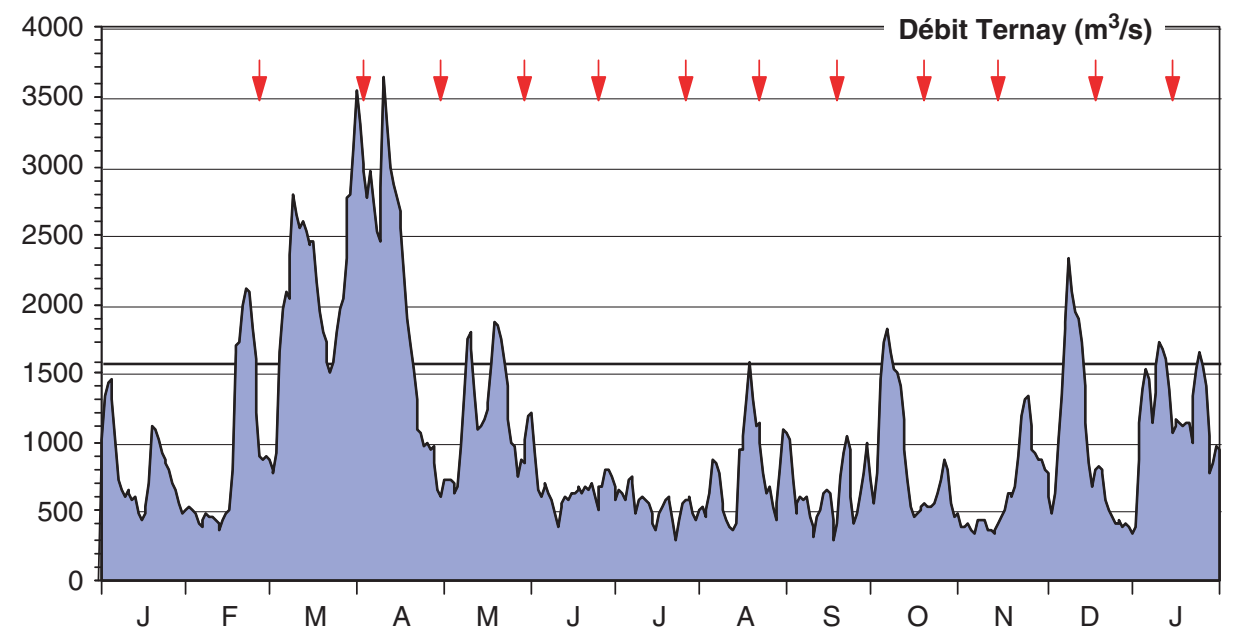

Fig. 3. Débits moyens journaliers du Rhône à Ternay durant la période d'étude janvier 2006 - janvier 2007 (données CNR Info Rhône). Le trait sombre horizontal représente la limite du débit d'armement du canal de dérivation ( $1600 \mathrm{~m}^{3} / \mathrm{s}$ ) au-delà de laquelle l'excédent de débit transite par la partie courtcircuitée. Les 12 dates de retrait des substrats artificiels sont figurées par une flèche.

Fig. 3. Daily medium discharge of the Rhône at Ternay gauging station during the study period January 2006 - January 2007 (data CNR Info Rhône). The horizontal dark line represents the limit of the maximum flow of the diversion canal $\left(1600 \mathrm{~m}^{3} / \mathrm{s}\right)$ beyond which the excess flow is discharged into the by-passed section. The 12 dates of withdrawal of the artificial substrates are illustrated by an arrow.

caniculaire (coefficient de 1,9 en avril vs. 0,6 en juillet). Elle est plus fluctuante sur la seconde partie d'été et le début de l'automne pour être à nouveau largement déficitaire au mois de novembre (coefficient de 0,6) avec la douceur climatique automnale qu'a connue l'année 2006. L'hydraulicité reste modérée en fin d'année et en début d'année suivante (coefficient de 0,8 en décembre et 0,9 en janvier).

\section{Température}

Des enregistrements en continu de la température locale de chaque substrat artificiel ont été réalisés à partir de capteurs VEMCO - Minilog-TR.
Ceux-ci étaient immergés à une profondeur comprise entre $1,50 \mathrm{~m}$ et $2 \mathrm{~m}$ au niveau des substrats artificiels. Ils sont restés en place de début mars 2006 à fin janvier 2007. Le pas de temps d'enregistrement est horaire. L'évolution temporelle de cette chronique est représentée sur la figure 4. Le tableau I présente un résumé des principales valeurs mesurées.

Ces données mettent principalement en évidence :

- Un écart de la température moyenne annuelle par rapport à la référence amont d'environ $4^{\circ} \mathrm{C}$ à l'aval et $1^{\circ} \mathrm{C}$ dans le RCC.

- Un minimum horaire jamais inférieur à $9^{\circ} \mathrm{C}$ en aval, soit supérieur 



Fig. 4. Enregistrements en continu de la température aux trois sites durant la période d'étude janvier 2006 - janvier 2007, exprimés en valeurs moyennes journalières, échauffement aux stations aval et RCC par rapport à l'amont (deltaT / amont) et amplitude thermique journalière.

Fig. 4. Recordings of the temperature to the three sampling sites during the study period January 2006 - January 2007, expressed in daily average values, temperature rise compared with upstream and daily thermal amplitude. 
Tableau I. Résumé statistique des enregistrements de température effectués du $1^{\text {er }}$ mars 2006 au 31 janvier 2007.

Table I. Statistical summary of the recordings of temperature carried out of from March 1st, 2006 to January 31, 2007.

\begin{tabular}{lcrr}
\hline $\mathbf{T}\left({ }^{\circ} \mathbf{C}\right)$ & Amont & Aval & RCC \\
\hline Nb de mesures journalières & 337 & 337 & 337 \\
Moyenne annuelle & 15,1 & 19,0 & 16,0 \\
Minimum journalier & 5,5 & 10,4 & 5,5 \\
Maximum journalier & 26,6 & 29,0 & 27,2 \\
Minimum horaire & 5,2 & 9,0 & 5,3 \\
Maximum horaire & 27,1 & 30,2 & 29,6 \\
Delta T / amont moyen & & 3,9 & 0,9 \\
Delta T / amont mini. & & 0,3 & 0 \\
Delta T / amont maxi. & & 7,3 & 3,3 \\
Amplitude journalière moy. & 0,8 & 1,9 & 2,1 \\
Amplitude journalière mini. & 0,1 & 0,4 & 0,2 \\
Amplitude journalière maxi & 5,0 & 5,5 & 5,7 \\
\hline
\end{tabular}

d'environ $4^{\circ} \mathrm{C}$ à celui enregistré aussi bien à l'amont que dans le RCC.

- Un maximum horaire voisin de $30^{\circ} \mathrm{C}$ lors de la canicule de début juillet 2006 aussi bien à l'aval que dans le RCC; il est de $27^{\circ} \mathrm{C}$ à l'amont.

- Un échauffement moyen par rapport à l'amont de $4^{\circ} \mathrm{C}$ à l'aval et voisin de $1^{\circ} \mathrm{C}$ dans le RCC (pouvant respectivement être en maximum horaire $>7^{\circ} \mathrm{C}$ et $>3^{\circ} \mathrm{C}$ ). D'un point de vue saisonnier, l'échauffement est compris entre 4 et $6^{\circ} \mathrm{C}$ aux intersaisons et entre 2 et $4^{\circ} \mathrm{C}$ en période chaude (printemps-été) à la station aval et dans la gamme de 2 à $4{ }^{\circ} \mathrm{C}$ toute l'année dans le RCC.

- Une amplitude journalière du même ordre à l'aval et dans le RCC, que ce soit en valeur moyenne $\left(2^{\circ} \mathrm{C}\right.$ environ à comparer à $<1^{\circ} \mathrm{C}$ à l'amont), qu'en valeur maximale (voisine de $5,5^{\circ} \mathrm{C}$ à comparer à $5^{\circ} \mathrm{C}$ à l'amont). Celle-ci est toutefois particulièrement marquée dans le RCC au printemps et en été, souvent comprise entre 4 et $5{ }^{\circ} \mathrm{C}$ lors des fortes températures de juin et juillet.

- Un franchissement du seuil printanier de $12^{\circ} \mathrm{C}$ (en valeur moyenne journalière) dès le début de nos enregistrements le $1^{\mathrm{er}}$ mars à la station aval et le 19 avril à l'amont et dans le RCC. Dès le 24 avril, la station aval franchit pratiquement le seuil estival de $18^{\circ} \mathrm{C}$, qui ne sera franchi que les 7 et 9 juin dans le $\mathrm{RCC}$ et à l'amont.

- Les trois stations passent pratiquement ensemble sous la barre des $18^{\circ} \mathrm{C}$ début octobre mais le seuil automnal de $12{ }^{\circ} \mathrm{C}$ est franchi les 22-23 novembre à l'amont et dans le RCC alors qu'à la station aval la température ne reste inférieure à $12^{\circ} \mathrm{C}$ que pendant une quinzaine de jours à partir de fin décembre jusqu'à fin janvier. 
- Le nombre de jours avec une température moyenne $\geq 23^{\circ} \mathrm{C}$ est de 88 jours à l'aval, soit un peu plus du double qu'à l'amont et dans le RCC (37 et 39 jours).

Les différents processus biologiques étant sous la dépendance de variables environnementales périodiques telles que la durée du jour ou la température, on imagine parfaitement l'importance que peuvent avoir les différences inter-stationnelles de ces divers seuils thermiques vis-àvis de la physiologie des organismes. Le début et la durée des différentes périodes thermiques auront donc une influence non négligeable sur le cycle de vie des organismes aquatiques ectothermes que sont les poissons et les invertébrés.

\section{4 ÉVOLUTION HISTORIQUE DES PEUPLEMENTS DE CRUSTACÉS}

Le tableau II synthétise les données concernant les crustacés recueillies au cours des suivis annuels du site de Saint-Alban aux trois stations considérées dans cette étude.

La figure 5 illustre l'évolution interannuelle de l'abondance moyenne par substrat artificiel unitaire des espèces de crustacés les plus abondamment représentées :

- d'une part deux Amphipodes natifs Gammarus pulex et Gammarus fossarum, l'Isopode Asellus aquaticus et l'écrevisse américaine Orconectes limosus tous deux apparus de longue date;

- d'autre part quatre espèces invasives plus récentes : les deux Gammaridés Gammarus tigrinus et Dikerogammarus villosus, ainsi que la crevette Atyaephyra desmarestii et l'Amphipode Chelicorophium curvispinum.

Cette figure met en évidence la décroissance progressive des premiers et l'apparition et l'extension des seconds. Dans le dernier cas, le parallèle peut être fait avec l'hydrologie, et en particulier les grands épisodes de crues (cf. § 3) qui accélèrent ces échanges, initialement favorisés par le développement des liaisons fluviales inter-bassins (Aron \& Smith, 1971; Jazdzewski, 1980 ; Bryson, 1992 ; Den Hartog et al., 1992 ; Vanden Bossche, 2002 ; Wouters, 2002 ; etc.).

Les Gammares natifs présentent une certaine concordance entre les années de présence plus abondante et les années de forte hydraulicité telles que 1987-88, 1994-(95), 1999, 2001-2002.

Bien que présentant des variations inter-annuelles classiques pour cette espèce à cycle de reproduction court et à fécondité importante (Henry \& Magniez, 1983), les effectifs d'A. aquaticus, plus faibles à la station aval depuis la fin des années 80 , sont en régression constante sur l'ensemble du secteur à partir de 2000 , et particulièrement bas lors des années avec une hydraulicité notablement faible comme 1996-97 et les 3 dernières années.

L'écrevisse américaine $O$. limosus est échantillonnée en petits nombres depuis le début du suivi, mais il semble que sa présence devienne plus régulière depuis 1997 dans l'ensemble des stations, avec une préférence pour la station aval « réchauffée ».

Les deux Gammaridés invasifs, G. tigrinus et $D$. villosus, sont récoltés régulièrement depuis 1999 et de façon 
Tableau II. Effectif total des différents taxons de crustacés échantillonnés par substrat artificiel rivulaire à Saint-Alban entre 1985 et 2006. Pour les espèces invasives, l'année d'apparition à Saint-Alban et l'origine sont précisées.

Table II. Total strength of different Crustaceans taxa sampled by riverine artificial substrate at SaintAlban between 1985 and 2006. For the alien species, the year of appearance at Saint-Alban and the origin are specified.

\begin{tabular}{|c|c|c|c|c|}
\hline $\begin{array}{l}\text { Crustacés Saint-Alban } \\
1985-2006\end{array}$ & Amont & Aval & RCC & $\begin{array}{c}\text { Origine des espèces } \\
\text { invasives }\end{array}$ \\
\hline $\mathrm{Nb}$ total de substrats & 97 & 98 & 96 & \\
\hline Hemimysis anomala & 1 & 0 & 1 & 2003 - Ponto-caspien \\
\hline Crangonyx pseudogracilis & 0 & 2 & 3 & 1995 - Am. Nord \\
\hline Gammarus fossarum & 617 & 126 & 485 & \\
\hline Gammarus pulex & 24 & 13 & 13 & \\
\hline Gammarus roeseli & 4 & 4 & 11 & < 1985 - Balkans \\
\hline Gammarus tigrinus & 194 & 360 & 27 & 1995 - Am. Nord \\
\hline Dikerogammarus villosus & 2133 & 634 & 1763 & 1999 - Ponto-caspien \\
\hline Gammarus sp. (juv.) & 404 & 111 & 599 & \\
\hline Gammaridae (juv.) & 689 & 97 & 310 & \\
\hline Chelicorophium curvispinum & 23 & 29 & 165 & 2002 - Ponto-caspien \\
\hline Asellus aquaticus & 77725 & 30834 & 20784 & Indét. - Sud-est Asie \\
\hline Proasellus meridianus & 1586 & 1831 & 832 & \\
\hline Asellidae juv. & 24377 & 5936 & 5044 & \\
\hline Orconectes limosus & 21 & 79 & 30 & < 1985 - Am. Nord \\
\hline Atyaephyra desmarestii & 59 & 1 & 19 & 2000 - Méditerranéen \\
\hline
\end{tabular}

NB : le nombre d'individus d'A. desmarestii est probablement sous-évalué en raison du caractère plutôt pélagique de cette espèce (crevette).

nettement plus abondante à partir de 2003, première année d'une période d'hydraulicité basse. Si la première espèce paraît particulièrement apprécier, au cours des deux dernières années, les eaux plus chaudes en aval de la centrale, c'est l'inverse pour le second taxon qui, dans la retenue a toujours été moins abondant en secteur réchauffé.

La crevette pélagique $A$. desmarestii et l'Amphipode filtreur vivant dans un tube limoneux fixé au substrat C. curvispinum semblent encore en phase d'installation et la tendance du premier à éviter le rejet de la centrale, et du second à préférer les eaux plus courantes du RCC demande à être confirmée.

\section{SUIVI MENSUEL 2006-2007}

Les résultats des 12 campagnes de prélèvements sont synthétisés dans le tableau III.

Toutes les espèces rencontrées au cours des suivis saisonniers des années précédentes ont été retrouvées lors du suivi mensuel de 2006, soit 12 espèces. Cependant, 4 taxons sont récoltés en quantités trop faibles pour donner lieu à une représentation graphique. Il s'agit de :

- la famille des Mysidés avec Hemimysis anomala, récolté en un seul exemplaire en amont de la retenue en novembre; 


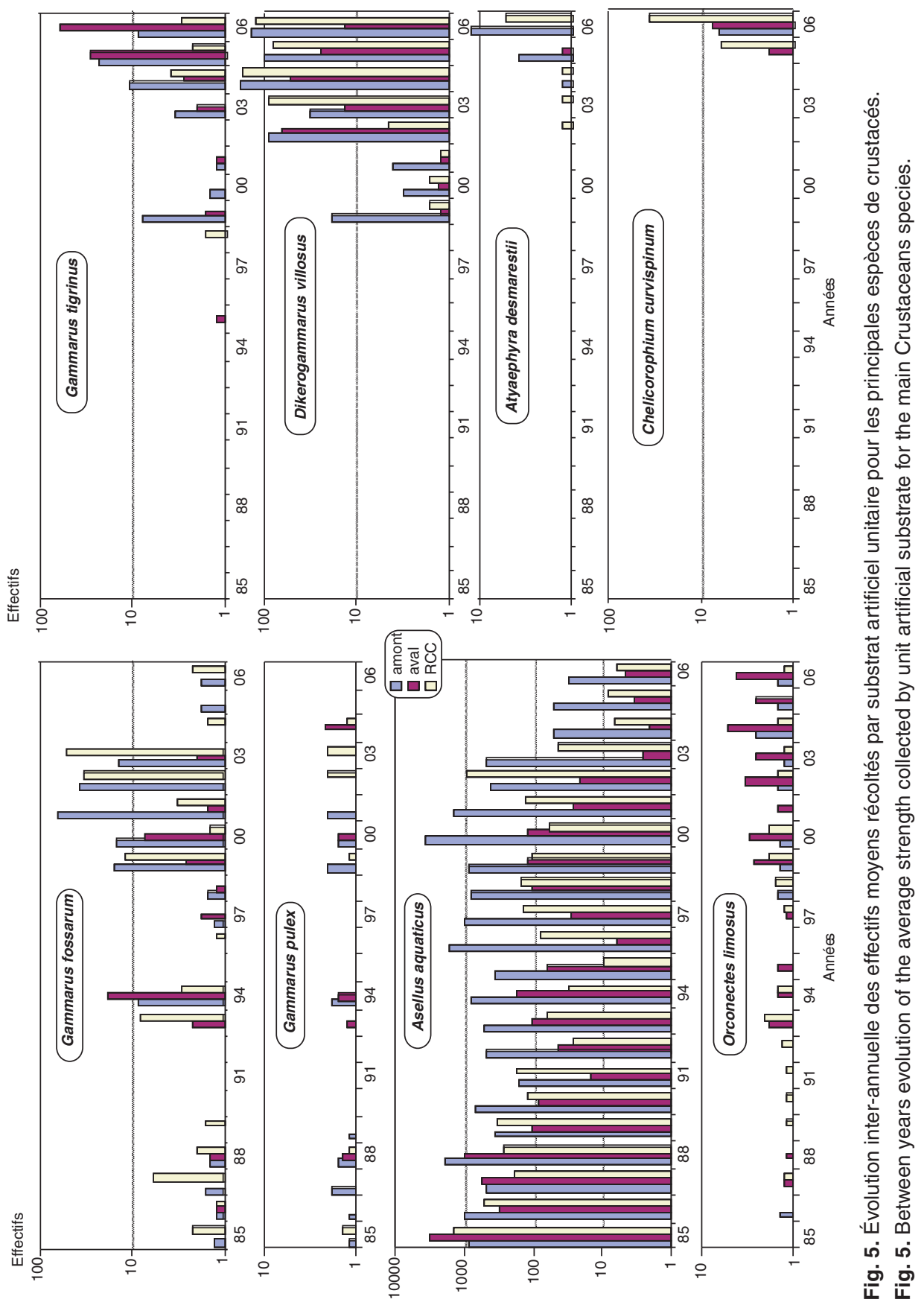


Tableau III. Synthèse des résultats enregistrés à chaque station d'échantillonnage exprimés par l'abondance totale et l'occurrence par campagne d'échantillonnage.

Table III. Summary of the results recorded at each sampling station (total abundance and occurrence per sampling campaign).

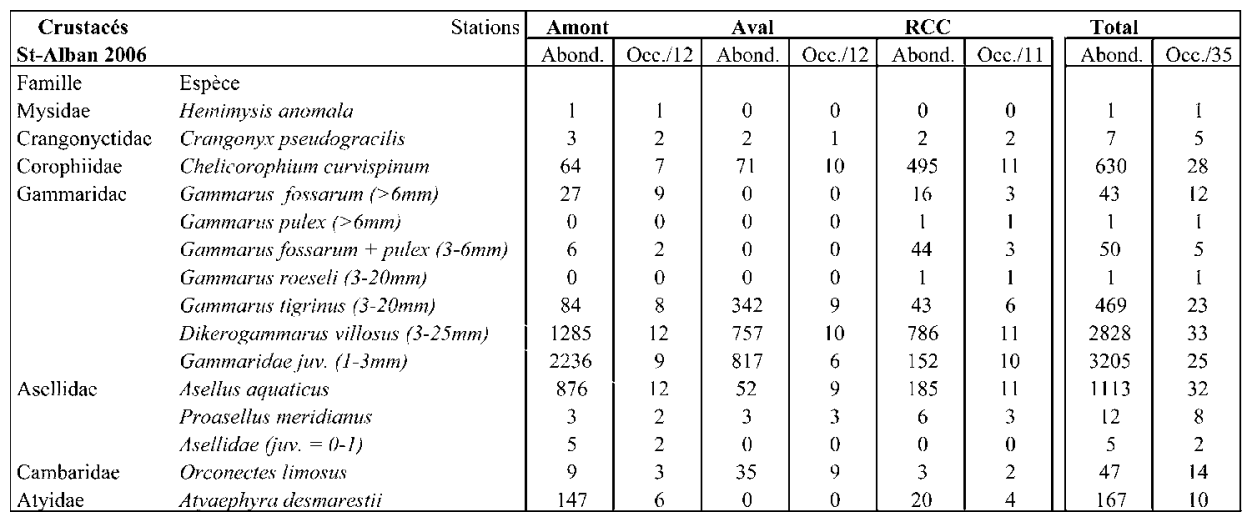

- la famille des Crangonyctidés avec Crangonyx pseudogracilis, rencontré à chaque station avec une occurrence et un effectif toutefois inférieurs à 2;

- l'espèce Gammarus roeseli avec un seul individu dans le RCC en juin (toujours plus abondante dans le plan d'eau de St-Pierre-deBœuf);

- enfin, le second représentant de la famille des Asellidés, Proasellus meridianus, qui ne compte que 12 individus.

\section{1 Étude globale du peuplement de crustacés}

Les effectifs de crustacés apparaissent nettement plus abondants à la station amont de la retenue qu'aux deux autres stations où les effectifs totaux sont assez proches (Fig. 6A). Ceci est essentiellement dû à une plus forte abondance d'Asellus aquaticus et des Gammaridae juvéniles, voire dans une moindre mesure de Dikerogammarus villosus (Tab. III).

Les proportions des diverses unités taxonomiques au sein de ces peuplements globaux sont sensiblement différentes entre les stations (Fig. 6B) :

- À l'amont, D. villosus avec les jeunes Gammaridae (probablement essentiellement des juvéniles de cette espèce dans ce cas) représentent globalement près de $75 \%$ des individus récoltés, $A$. aquaticus approche encore les $20 \%$, puis viennent ensuite $A$. desmarestii, $G$. tigrinus et $C$. curvispinum.

- À la station aval, G. tigrinus est numériquement et proportionnellement $(16 \%)$ plus abondant qu'à l'amont, alors que $D$. villosus (36\%) et les juvéniles de cette famille (39\%), bien que moins nombreux, sont également proportionnellement plus abondants qu'à 


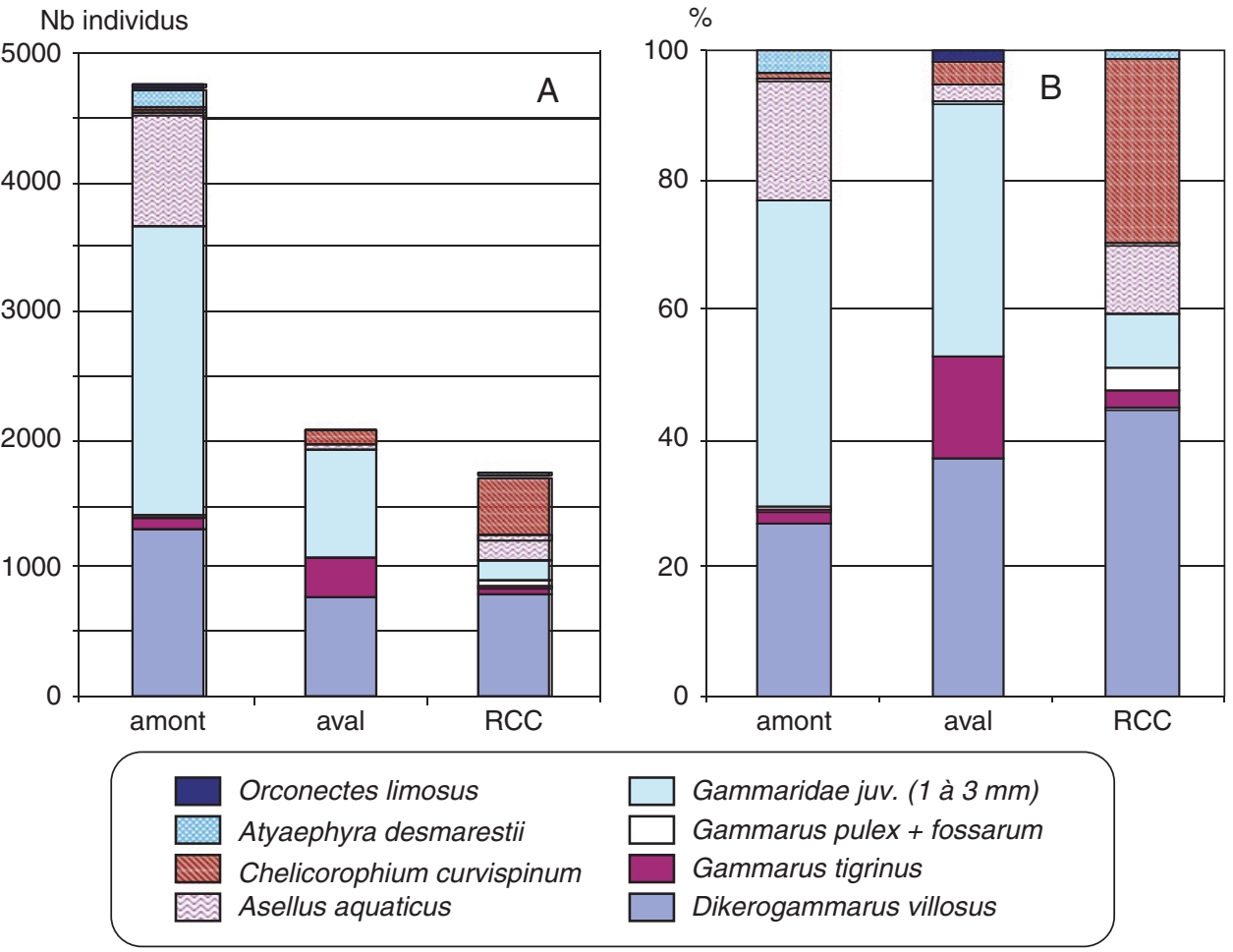

Fig. 6. Effectifs totaux (A) et abondances relatives totales (B) des principaux crustacés récoltés aux trois stations.

Fig. 6. Total strength (A) and total relative abundances (B) of main Crustaceans species collected to the three sampling stations.

la station de référence. $A$. aquaticus $(2,5 \%)$ est dépassé par $C$. curvispinum et suivi par l'écrevisse $O$. limosus.

- Dans le secteur court-circuité, l'abondance relative de $D$. villosus augmente sensiblement (45\%), suivi de $C$. curvispinum (28\%) et A. aquaticus $(11 \%)$. À cette station, la plus courante des trois, les Gammares autochtones (G. pulex et $G$. fossarum) apparaissent significatifs avec 3,5\% des captures de crustacés, alors qu'ils sont anecdotiques en amont de la retenue $(0,7 \%)$ et absents en aval.

Les évolutions temporelles des effectifs des divers taxons suivent un schéma différent selon les stations (Fig. 7A). En raison de ses trop faibles effectifs par échantillon, l'écrevisse américaine $O$. limosus n'a pas été représentée. Toutefois, ses effectifs les plus élevés (une dizaine d'individus 
$\mathrm{Nb}$ individus
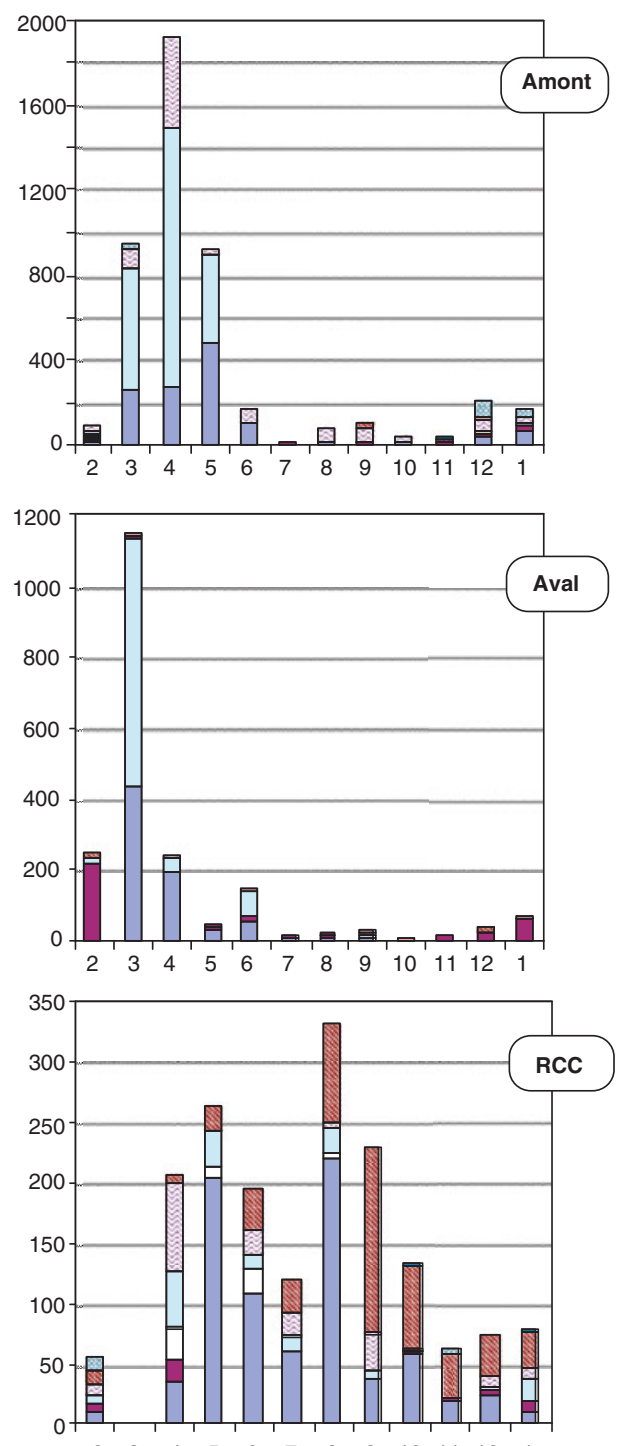

$\%$ B
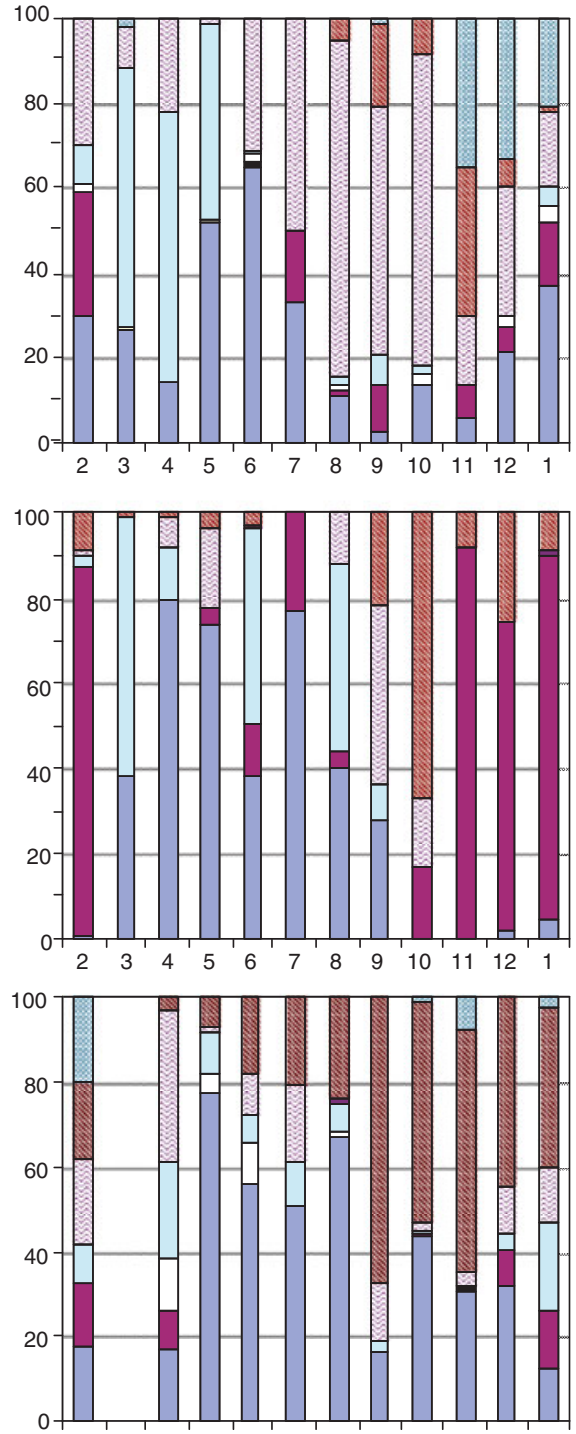

$\begin{array}{llllllllllll}2 & 3 & 4 & 5 & 6 & 7 & 8 & 9 & 10 & 11 & 12 & 1\end{array}$ mois 2006-2007

$\square$ Atyaephyra desmarestii $\square$ Gammaridae juv. (1 à $3 \mathrm{~mm}$ ) $\square$ Gammarus tigrinus
Chelicorophium curvispinum $\square$ Gammarus pulex + fossarum $\square$ Dikerogammarus villosus
Asellus aquaticus

Fig. 7. Évolution temporelle des effectifs (A) et des abondances relatives (B) des principaux crustacés récoltés aux trois stations. NB : en raison de ses trop faibles effectifs par échantillon, l'écrevisse O. limosus n'est pas représentée.

Fig. 7. Temporal evolution of strength (A) and relative abundances (B) of main Crustaceans species collected to the three sampling stations. NB: because of its too weak strength by sample, the crayfish $O$. limosus is not represented. 
par substrat artificiel) ont été notés à la station aval au mois de mai et juin.

Faibles en début d'année (fin février) quelle que soit la station, les effectifs sont maximum au printemps dans la retenue avec un mois d'avance dans la station aval réchauffée (début avril) par rapport à l'amont (début mai). On remarque également qu'en aval de la centrale les effectifs, globalement plus faibles (cf. précédemment), présentent un pic plus étroit : une seule date avec plus de 800 individus contre trois dates en amont. Dès le mois de mai à la station aval, puis à partir de juin en amont, les effectifs sont bas et le demeurent jusqu'à la fin de l'année. Dans le RCC, les crustacés, bien que moins nombreux que dans la retenue, montrent une meilleure répartition tout au long du cycle annuel.

Dans la retenue, les premiers échantillons des 2 stations (Fig. 7B) sont caractérisés par la présence de G. tigrinus (respectivement $20 \%$ et plus de $80 \%$ en amont et en aval), qui devient ensuite quasi absent lors des 4 campagnes suivantes (début avril à fin juin), peut-être éliminé par la longue période de hautes eaux enregistrée de début mars à fin avril. On le retrouve par la suite plus ou moins régulièrement aux 2 stations, mais surtout en aval du rejet où il approche et dépasse $80 \%$ lors des 3 dernières campagnes. $D$. villosus, associé au printemps aux juvéniles de la même famille, dominent en amont jusqu'au milieu de l'été, relayé de juin à octobre par Asellus aquaticus, lui-même relayé d'août à décembre par $C$. curvispinum puis par la crevette $A$. desmarestii en fin de cycle (novembrejanvier). En aval du rejet, les effectifs sont si faibles de juillet à la fin du cycle d'échantillonnage que les proportions sont très peu significatives, à part le retour de $G$. tigrinus en fin d'année.

Dans le RCC, Chelicorophium curvispinum renforce son importance au fil des prélèvements au détriment apparent de Dikerogammarus villosus, les Aselles se maintenant au fil du temps. Les deux Gammares du Haut-Rhône G. fossarum et G. pulex sont échantillonnés pendant les deux mois suivant la période de hautes eaux.

Une analyse factorielle des correspondances inter-classes (Dolédec \& Chessel, 1989) a été réalisée sur le tableau de données (35 relevés unitaires x 12 taxons) afin de synthétiser les informations précédentes (Fig. 8). Les calculs ont été effectués à l'aide du logiciel ADE-4 (Thioulouse et al., 1997).

L'analyse inter-dates présente un cycle temporel parfait de la fin de la saison froide 2006 (mars - partie positive de l'axe 1) à la saison froide 2007 (janvier-février - partie négative de l'axe 1), plus particulièrement décrit par l'abondance des jeunes stades de Gammaridae (G1 et G2) en hiver et au printemps 2006 et par l'accroissement des effectifs de $G$. tigrinus et $C$. curvispinum fin 2006 et hiver 2007 (Figs. 8B et $8 \mathrm{C})$.

L'analyse inter-stations oppose, d'une part, la station aval réchauffée aux deux autres stations sur l'axe 1 (limitation des effectifs généraux à la station aval mais effectifs particuliers toutefois plus élevés pour G. tigrinus et $O$. limosus) et, d'autre part, les stations de la retenue au RCC sur l'axe 2 (abondance notable de C. curvispinum dans la partie courtcircuitée) (Figs. 8E et 8F). 

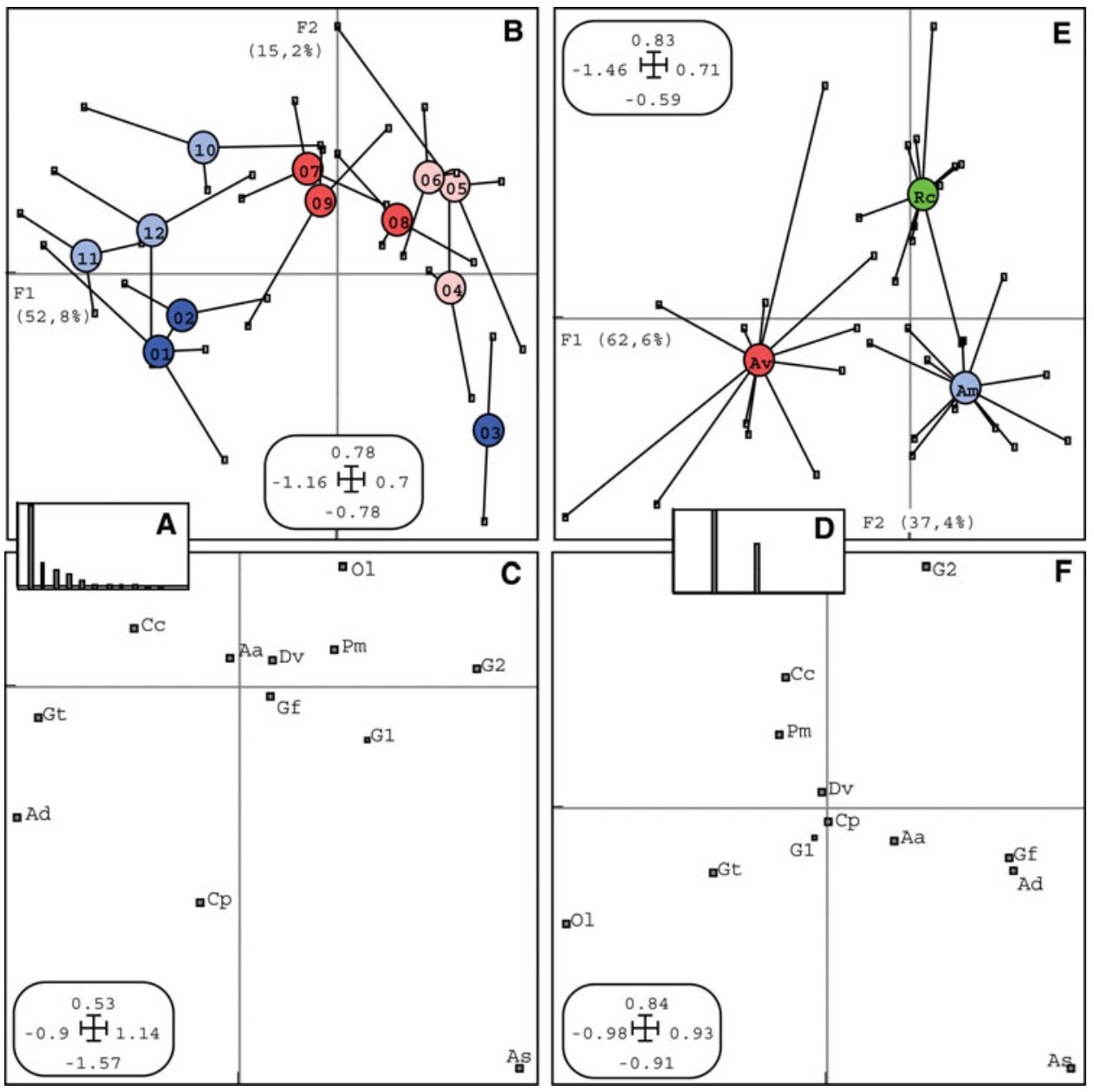

Fig. 8. Analyse inter-classes des peuplements de crustacés. AFC inter-dates : (A) Graphe des valeurs propres, (B) Carte factorielle $1 \times 2$ des relevés (dates), (C) Carte factorielle $1 \times 2$ des espèces. AFC interstations : (D) Graphe des valeurs propres, (E) Carte factorielle 1x2 des relevés (stations), (F) Carte factorielle $1 \times 2$ des espèces. Dates $=$ mois d'immersion des substrats artificiels. Code des taxons : $\mathrm{Cp}$ Crangonyx pseudogracilis, Cc Chelicorophium curvispinum, Gf Gammarus fossarum, Gt Gammarus tigrinus, Dv Dikerogammarus villosus, G1 Gammaridae juvéniles 1-3 mm, G2 Gammaridae juvéniles 3-6 mm, Aa Asellus aquaticus, Pm Proasellus meridianus, As Asellidae juvéniles, Ol Orconectes limosus, Ad Atyaephyra desmarestii.

Fig. 8. Between-class analysis of Crustaceans communities. Between-dates CA: (A) eigenvalues graph, (B) samples (dates) factorial map 1×2, (C) species factorial map 1×2. The circles correspond to the centre of classes and the lines correspond to the dispersion of samples. Between-stations CA: (D) eigenvalues graph, (E) samples (stations) factorial map 1x2, (F) species factorial map 1x2. 


\section{2 Étude spécifique}

Asellus aquaticus, initialement originaire du sud-est de l'Asie, est présent depuis très longtemps en France (Henry \& Magniez, 1983). D'une durée de vie brève (en général $<1$ an, parfois 18 mois), on trouve des femelles ovigères durant les trois-quarts de l'année, avec une période de reproduction massive au printemps. Avec une fécondité considérable (souvent plus de 100 œufs par ponte) et un développement marsupial très rapide (15 jours environ), l'abondance des populations d'Aselles est soumise à une variation annuelle cyclique très marquée (Henry \& Magniez, op. cit.).

À Saint-Alban, les effectifs d'Aselles, dont la diminution est constatée depuis le début des années 2000 (Bady \& Fruget, 2006) aux 2 stations de la retenue et principalement en aval du rejet, sont au cours des 6 premiers mois 2006 à nouveau très inférieurs en aval de la centrale. Début mai, le pic d'abondance (Fig. 9) lié à la reproduction noté en amont de la retenue (99\% d'individus < $6 \mathrm{~mm}$ ) n'est pas visible en aval de rejet (où $100 \%$ des individus sont $<6 \mathrm{~mm}$ ). À la fin du mois, ce taxon est en nette régression aux 2 stations et il ne subsiste une modeste population qu'en amont de la retenue alors que sous l'influence de la centrale les effectifs capturés ne dépassent la dizaine qu'à 2 dates et sont nuls à 3 reprises.

On note également que, contrairement à ce qui est observé en amont, la grande majorité des quelques individus récoltés en aval du rejet est constituée de juvéniles tout au long de l'année (Fig. 10B), ce qui peut correspondre soit à une mortalité rapide des jeunes adultes rencontrés en début de cycle à cette station, soit à un apport par dérive de jeunes individus depuis l'amont. Dans le secteur court-circuité la population est également très limitée, exception faite du début du mois de mai, mais le caractère nettement plus lotique de cette station est un facteur limitant attendu pour cette espèce de milieux plutôt calmes. L'évolution temporelle des pourcentages de jeunes individus du RCC est très proche de celle observée à la station de référence.

Dans la retenue, l'occurrence de l'Amphipode filtreur Ponto-Caspien Chelicorophium curvispinum, qui s'abrite dans des tubes limoneux sur les substrats durs et se nourrit de phytoplancton et de sédiments fins (Van den Brink et al., 1991; Genin, 1992), est en début d'année bien supérieure en aval du rejet, les effectifs les plus élevés correspondant, à cette station, à la saison froide. Après une période d'équivalence durant la première partie de la période estivale, on observe le phénomène inverse d'août à novembre avec des effectifs plus importants à l'amont.

À partir de la fin du mois de mai, la population la plus nombreuse est notée dans le RCC avec un pic d'abondance s'étalant d'août à octobre, correspondant à la fin de sa période de reproduction (avril-octobre), et par ailleurs de stabilité hydraulique. Malgré des niches écologiques sensiblement différentes, certains auteurs considèrent cette espèce comme pouvant rentrer en compétition avec A. aquaticus (Bij de Vaate et al., 2002).

La crevette pélagique Atyaephyra desmarestii, d'origine méditerranéenne, absente du Haut-Rhône mais bien présente dans la végétation des 

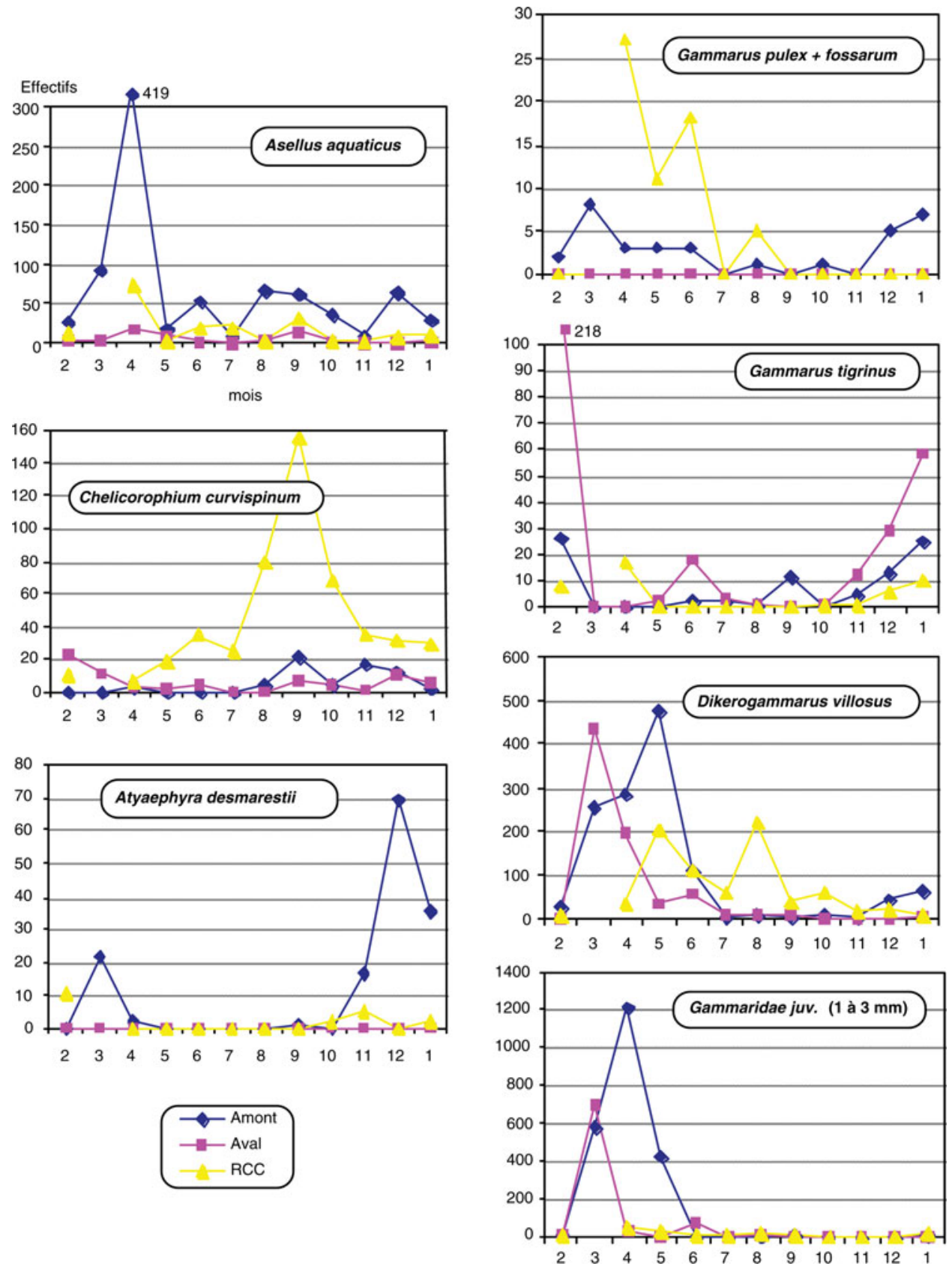

Fig. 9. Évolution temporelle des effectifs récoltés lors des 12 campagnes d'échantillonnage pour les principales espèces de crustacés.

Fig. 9. Temporal evolution of the strength collected during the 12 sampling campaigns for the main Crustaceans species. 


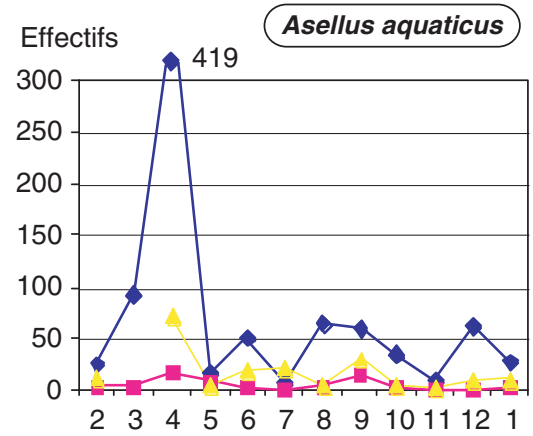

$\%$ juvéniles

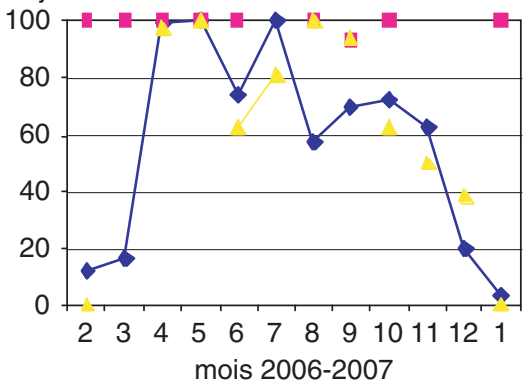

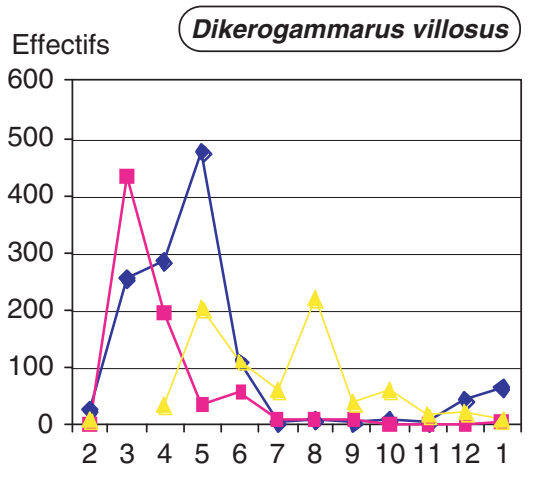

$\%$ juvéniles

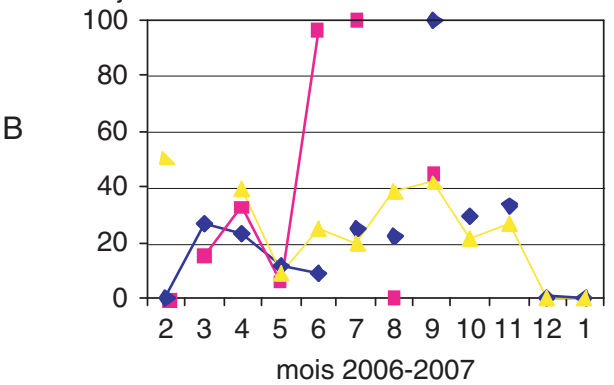

Fig. 10. Évolution temporelle des effectifs totaux récoltés $(A)$ et des pourcentages de juvéniles $(B)$ pour les deux taxons les plus abondants. Les points isolés ou reliés par des pointillés correspondent à de très faibles effectifs.

Fig. 10. Temporal evolution of the collected total strength $(A)$ and the percentages of juveniles (B) for both most abundant taxa. The points isolated or connected by dotted lines correspond to very weak strength.

rives calmes de la Saône, n'est trouvée dans notre secteur d'étude qu'en début d'année 2006 (fin février - début mai) et en automne et fin d'année (octobre à janvier 2007) après une absence presque totale (de nos relevés, car régulièrement capturée lors des pêches électriques intermédiaires) de 5 mois. II se peut que l'absence de végétation en automne-hiver facilite sa capture par substrat artificiel qui constitue alors un refuge où elle est piégée. Dans la retenue, elle marque une nette préférence pour la station amont et elle est totalement absente à l'aval. Son abondance est moindre dans le RCC.

Les deux espèces de Gammares rhéophiles, autochtones (et typiques des eaux relativement fraîches du Haut-Rhône) que sont Gammarus pulex et Gammarus fossarum sont classiquement peu présentes dans l'ensemble du secteur. C'est logiquement dans le RCC (Fig. 9) qu'elles sont plus présentes, et plus particulièrement à la suite de forts débits. On note que si quelques rares individus 
peuvent se rencontrer en amont de la retenue, ces deux espèces sont absentes tout au long du cycle annuel en aval du rejet.

D'origine Nord-Américaine, Gammarus tigrinus est sans doute le crustacé du secteur que la littérature considère comme le plus tolérant aux températures élevées en été et aux rapides réchauffements printaniers (Hynes, 1955 ; Van Maren, 1978; par ex.) mais, inversement, régressant fortement lors d'hivers froids (Pinkster et al., 1980). Fin février, ce taxon est 8 fois moins abondant en amont que dans le secteur réchauffé en aval de la centrale. À cette station, $80 \%$ des femelles sont ovigères contre seulement $61 \%$ à la station de référence amont. Lors de la campagne suivante, ce taxon disparaît de la retenue où il n'est à nouveau significativement présent que fin juin en aval. Les forts débits de la fin de l'hiver et du début du printemps peuvent expliquer cette absence, ce taxon résistant mal à des vitesses de courant trop élevées (Nijssen et al., 1966). Dans le RCC, présent en faible quantité en février, on le retrouve début mai, puis il disparaît lors des 5 campagnes suivantes. Lors des derniers échantillonnages, les effectifs augmentent régulièrement aux 3 stations, mais plus sensiblement là où le courant est le plus faible et la température la plus élevée.

Le taxon invasif Ponto-Caspien Dikerogammarus villosus est décrit par de très nombreux auteurs comme le plus prédateur des représentants de la famille des Gammaridés (Bij de Vaate et al., 2002; Dick et al., 2002), étant parfois même qualifié d'«espèce tueuse » pour les autres taxons de sa propre famille (Vanden Bossche, 2002) en raison notamment d'une taille maximum bien supérieure, d'une fécondité élevée et d'une croissance rapide (Devin et al., 2004). II lui est de ce fait également souvent attribué un fort impact sur les populations de G. tigrinus (Bij de Vaate et al., 2002).

Dans notre secteur d'étude, $D$. villosus est échantillonné en petits nombres en hiver aux 3 stations. Dans la retenue, ses effectifs augmentent fortement dès le début du printemps. Pour la population de la station de référence amont, l'augmentation d'abondance s'étale sur les 3 campagnes printanières alors que celle du secteur réchauffé enregistre son maximum d'effectif début avril, soit 2 mois plus tôt, puis diminue fortement. En début d'été la population amont régresse à son tour, pour redescendre fin juillet à de faibles effectifs, proches de ceux du secteur réchauffé où il devient rare ou absent à partir de juillet alors qu'on le retrouve à la station de référence amont en décembre et janvier.

Dans le RCC, la population, plus limitée que dans la retenue jusqu'en juin, est au contraire plus conséquente de juillet à novembre.

Il faut noter en dernier lieu que la forte augmentation de $D$. villosus dans la retenue coïncide exactement avec la disparition à ces stations de G. tigrinus dont la présence ne redevient notable à l'aval que 2 mois après la régression en ce point de la première espèce. Ces deux taxons montrent également une évolution temporelle inverse dans le RCC, confirmant ainsi une possible compétition de ces deux invasifs à qui la littérature donne respectivement l'avantage selon le caractère lotique (i.e. courant) du peuplement pour le premier ou au contraire 
lentique (i.e. d'eau calme) pour le second.

La comparaison de l'évolution des tailles maximales des deux sexes (Fig. 11) aux deux stations de la retenue semble indiquer une mortalité en secteur réchauffé plus précoce (mai au lieu de juin) et plus intense des individus de grande taille ayant passé l'hiver (surtout des femelles). À partir de juillet, les effectifs sont trop faibles pour être interprétables. Dans le RCC, il est possible de déceler dans une population plus importante deux périodes de rajeunissement des adultes: mai-juin et septembre-octobre.

Taille en $\mathrm{mm}$

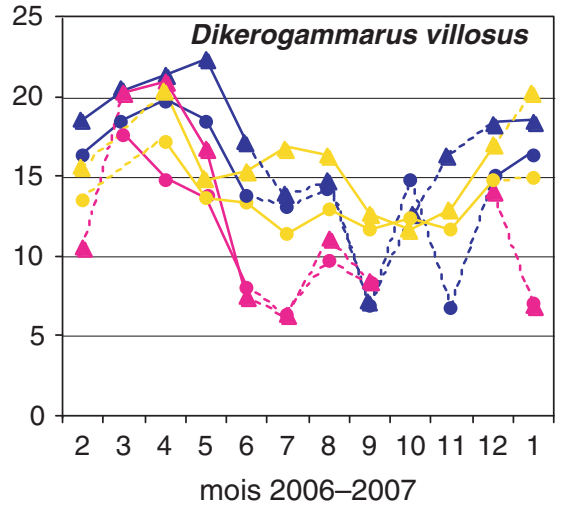

$\begin{array}{lll}\longrightarrow \text { M amont } & - \text { F amont } & - \text { M aval } \\ \longrightarrow \text { F aval } & - \text { M RCC } & - \text { F RCC }\end{array}$

Fig. 11. Évolution temporelle des tailles maximales des mâles et des femelles de Dikerogammarus villosus aux trois stations. Les points isolés ou reliés par des pointillés correspondent à de très faibles effectifs.

Fig. 11. Temporal evolution of the maximum sizes of the males and the females of Dikerogammarus villosus at the three sampling stations. The points isolated or connected by dotted lines correspond to very weak strength.
La variation temporelle de la catégorie Gammaridae indéterminables (taille < à $3 \mathrm{~mm}$ ), dont il est raisonnable d'estimer qu'elle est majoritairement (surtout au printemps dans la retenue) constituée de Dikerogammarus villosus confirme une reproduction plus précoce, plus intense, mais de plus courte durée dans la station réchauffée par le rejet de la centrale. Fin juin, à cette station, les très jeunes individus à nouveau présents peuvent correspondre à une deuxième vague de reproduction de $D$. villosus et/ou à de très jeunes $G$. tigrinus. Tout au long de l'année dans le secteur courtcircuité, ainsi que dans la retenue à partir de juillet, les individus les plus jeunes sont très peu nombreux. Ceci demeure difficilement explicable pour des taxons dont la reproduction devrait s'étaler sur une grande partie du cycle annuel : prédation par les plus gros individus et/ou milieu non conforme à leurs exigences?

\section{CONCLUSIONS}

L'étude de la chronique de température depuis le début du suivi au milieu des années 1980 met en évidence une dérive des températures sur la période considérée, parallèlement à d'autres événements ponctuels importants tels que les épisodes de crue et de canicule. Une hausse progressive des températures moyennes annuelles a ainsi été notée depuis le milieu des années 1990, s'accentuant sur les dernières années de la chronique : la moyenne mobile sur 5 années passe ainsi de $13,6^{\circ} \mathrm{C}$ en 1995 à $14,4^{\circ} \mathrm{C}$ en 2006.

Hormis l'amplitude journalière et les valeurs maximales, le fonctionnement 
thermique du Rhône en amont du CNPE est très similaire à celui de la partie court-circuitée. À l'opposé, la station aval présente un décalage notable par rapport à ces deux stations dans le sens d'une précocité : la température moyenne journalière y passe ainsi extrêmement rarement sous le seuil $12^{\circ} \mathrm{C}$; quand les stations amont et RCC franchissent ce seuil printanier, la station aval franchit le seuil $18^{\circ} \mathrm{C}$; la durée de températures moyennes journalières $\geq 23^{\circ} \mathrm{C}$ est plus du double à l'aval (88 jours) par rapport aux deux autres sites (< 40 jours), etc. On aboutit ainsi à la station réchauffée à une précocité des différents seuils thermiques et à un raccourcissement de la période froide.

Du point de vue biologique, la chronique de suivi du CNPE de Saint-Alban a permis d'enregistrer une première vague d'invasions parmi les crustacés (Gammarus tigrinus, Dikerogammarus villosus) durant les années 1990 sur le Rhône Moyen, principalement en relation avec d'importants épisodes hydrologiques qui accélèrent ces échanges, initiés à l'origine par le développement des liaisons fluviales inter-bassins. La période de canicule et post canicule du début des années 2000 se traduit ensuite par le développement d'une seconde vague d'espèces exotiques invasives (Atyaephyra desmarestii, Hemimysis anomala, Chelicorophium curvispinum) et l'installation durable des premiers arrivants.

Ainsi, dans le contexte général de changement climatique, les événements hydroclimatiques extrêmes (crues, canicule) ont favorisé l'installation de taxons de crustacés invasifs ubiquistes et résistants, le plus souvent thermophiles et potamo-lénitiques
(Dikerogammarus post 2001, Atyaephyra desmarestii et Hemimysis anomala post canicule). Ceci est également valable à l'échelle du peuplement d'invertébrés pris dans sa globalité avec l'apparition de Corbicula fluminea (Mollusque Bivalve) post 1993, et d'Hypania invalida (Polychète) post 2003 parmi les espèces invasives, mais aussi le développement du Trichoptère Ecnomus tenellus sur le Bas-Rhône, de la Planaire Dugesia tigrina et du Mollusque Gastéropode Potamopyrgus antipodarum sur le Haut-Rhône parmi les taxons potamo-lénitiques (Daufresne et al., 2003 et 2007; Bady \& Fruget, 2006). Plus généralement, ce type d'événements extrêmes provoque le développement d'espèces ubiquistes, tolérantes et exotiques qui tirent avantage de l'évolution des conditions environnementales et thermiques. Un phénomène similaire a parallèlement été mis en évidence à la même période sur la Meuse (Pinkster et al., 1992), le Rhin et la Moselle (Bij de Vaate, 1993, 1994 ; Bachmann et al., 1997; Kelleher et al., 1998) avec l'augmentation de l'eutrophisation et/ou des concentrations en chlorures et de la température moyenne de l'eau.

Le suivi spécifique mensuel durant un an des populations de crustacés a permis de préciser les points suivants :

- augmentation des effectifs hivernaux pour certaines espèces, Chelicorophium curvispinum et Gammarus tigrinus en particulier;

- limitation de certaines populations à la station aval réchauffée (Asellus aquaticus, Gammarus pulex, Gammarus fossarum, Atyaephyra desmarestii); 
- modification du cycle biologique : reproduction plus précoce mais de plus courte durée de Dikerogammarus villosus dans le secteur aval ;

- probabilité d'existence de phénomènes de compétition interspécifique (Gammarus tigrinus et Dikerogammarus villosus).

Les évolutions temporelles des effectifs des divers taxons suivent un schéma différent selon les stations. Faibles en début d'année (fin février) quelle que soit la station, les effectifs sont maximum au printemps dans la retenue avec un mois d'avance dans la station aval réchauffée (début avril) par rapport à l'amont (début mai). On remarque également qu'en aval de la centrale ces effectifs, globalement plus faibles, présentent un pic plus étroit : une seule date avec plus de 800 individus contre trois dates en amont.

Les effectifs limités, voire nuls, de certains taxons, essentiellement natifs $(A$. aquaticus, $G$. pulex et $G$. fossarum) dans le secteur réchauffé confirme des observations effectuées par ailleurs :

- seuil critique expérimental de $23^{\circ} \mathrm{C}$ pour les Aselles au-delà duquel, après une première ponte normale, les suivantes diminuent rapidement avec quelques œufs au lieu de plusieurs dizaines, voire absence d'œuf dans la poche incubatrice (Balesdent, 1965) ;

- baisse des effectifs de Gammares indigènes du Rhône à Bugey quand la température estivale est $>20^{\circ} \mathrm{C}$ et disparition de ceux-ci lorsqu'elle dépasse $25^{\circ} \mathrm{C}$ (Roger \& Faessel, 1989).
La variation temporelle de la catégorie Gammaridae indéterminables (jeunes individus de taille $<3 \mathrm{~mm}$ ), dont il est raisonnable d'estimer qu'elle est majoritairement (surtout au printemps dans la retenue) constituée de Dikerogammarus villosus confirme, pour ce taxon précis, une reproduction plus précoce, plus intense, mais de plus courte durée dans la station réchauffée par le rejet de la centrale.

D'un point de vue méthodologique, l'échantillonnage par substrats artificiels n'a pas permis de récolter assez régulièrement des quantités suffisantes des trois espèces (Asellus aquaticus, Dikerogammarus villosus, Gammarus tigrinus) dont on voulait initialement étudier en détails la dynamique de population; il faut en effet des effectifs > 100 individus pour déterminer la répartition en diverses catégories : juvéniles, mâles, femelles ovigères ou non et $>300$ individus pour effectuer la répartition en classes de tailles. Dans ce but, il faudrait tenter de compléter l'échantillonnage quantitatif par une récolte manuelle au filet de type Surber, suffisante pour atteindre ces effectifs.

Enfin, afin d'apprécier les modifications des interactions biotiques engendrées par les stress environnementaux que sont la variabilité hydroclimatique et les invasions biologiques, des études plus poussées faisant appel d'une part à la biologie des populations (structure génétique, traits d'histoire de vie, comportement prédateur, compétition inter-spécifique, fécondité taille dépendante, etc.) au travers d'expérimentations de laboratoire en conditions contrôlées, d'autre part à l'écologie évolutive afin 
d'apprécier les réponses aux contraintes de l'environnement (potentiel adaptatif des individus à la thermie en particulier) s'avèrent nécessaires.

\section{RÉFÉRENCES BIBLIOGRAPHIQUES}

ARALEP, 2003. Suivi hydrobiologique du Rhône au niveau de la centrale nucléaire de Saint-Alban - Saint-Maurice. Synthèse 1985-2001. Rapport à EDF St-Alban, 58 pp.

Aron W.I. \& Smith S.H., 1971. Ship canals and aquatic ecosytems. Science 174 : 13-20.

Bachmann V., Usseglio-Polatera P., Cegielka E., Wagner P., Poinsaint J. \& J.C. Moreteau, 1997. Premières observations sur la coexistence de Dreissena polyorpha, Corophium curvispinum et Corbicula spp. dans la Moselle. Bull. Fr. Pêche et Piscic. 344-345 : 373-384.

Bady P. \& Fruget J.F., 2006. Etude thermique globale du Rhône. Phase 3. Influence de la variabilité hydroclimatique 1985-2004 sur les peuplements de macroinvertébrés. Rapport conjoint ARALEP - LEHF Université Lyon 1 à EDF DTG, 30 pp.

Balesdent M.L., 1965. Recherche sur la sexualité et le déterminisme des caractères sexuels d'Asellus aquaticus. Bull. acad. Soc. Lorraine sci. 5 (2) : 1-231.

Bij de Vaate A., Jazdzewski K., Ketelaars H.A.M., Gollasch S., Van Der Velde G., 2002. Geographical patterns in range extension of Ponto-Caspian macroinvertebrate species in Europe. Can. J. Fish. Aquat. Sci. 59 : 1159-1174.

Bij de Vaate A., 1994. Long-term changes in the macroinvertebrate fauna of the River ljssel, The Netherlands. Vehr. Internat. Verein. Limnol. 25 (3) : 15631567.
Bij de Vaate A., 1993. Exotic and aquatic macroinvertebrates in the Dutch part of the River Rhine : causes and effects. In Ecological rehabilitation of the rivers Rhine and Meuse. Van Dijk G.M. \& Marteijn E.C.L. (Eds), Institute for Inland Water Management and Waste Water Treatment, Lelystad : 27-29.

Bryson B., 1992. Main-Danube Canal : Linking Europe's waterways. National Geographic, August 1992 : 3-31.

Carrel G., 2006. Etude thermique globale du Rhône. Phase III. Le régime thermique du fleuve. Variabilité temporelle 1977-2004. Rapport CEMAGREF Aixen-Provence à EDF DTG, 13 pp.

Daufresne M., Bady P. \& Fruget J.F., 2007. Impacts of long-term changes in temperature and extreme hydro-climatic events on macroinvertebrate community structures in the French Rhône River. Oecologia, Special issue on the impact of climate change on aquatic ecosystems, 151 : 544-559.

Daufresne M., Roger M.C., Capra H. \& Lamouroux N., 2004. Long-term changes within the invertebrate and fish communities of the Upper Rhone River : effects of climatic factors. Global Change Biology 10 : 124-140.

Den Hartog C., Van den Brink F.W.B. \& Van der Velde G., 1992. Why was the invasion of the river Rhine by Corophium curvispinum and Corbicula species so successful? J. Natur. Hist. 26 : 1121-1129.

Devin S., Piscart C., Beisel J.N., Moreteau J.C., 2004. Life history traits of the invader Dikerogammarus villosus (Crustacea, Amphipoda) in the Moselle River, France. Internat. Rev. Hydrobiol. 89 (1) : 21-34.

Dick J., Platvoet D. \& Kelly D., 2002. Predatory impact of the freshwater invader Dikerogammarus villosus (Crustacea : Amphipoda). Can. J. Fish. Aquat. sci. $59: 1078-1084$. 
Dolédec S. \& Chessel D., 1989. Rythmes saisonniers et composantes stationnelles en milieu aquatique. II. Prise en compte et élimination d'effets dans un tableau faunistique. Acta Oecol., Oecol. Gener. 10 (3) : 207-232.

Fruget J.F., Centofanti M., Dessaix J., Olivier J.M., Druart J.C. \& P.J. Martinez, 1999. Synthèse des dix premières années de suivi hydrobiologique du Rhône au niveau de la centrale nucléaire de Saint-Alban. Hydroécol. Appl. 11 (1-2) : 29-69.

Genin B., 1992. Corophium curvispinum Sars, 1895 (Crustacea, Amphipoda) dans la Saône. Bull. Sci. Bourg. 45 : 27-32.

Henry J.P. \& Magniez G., 1983. Introduction pratique à la systématique des eaux continentales françaises. 4. Crustacés Isopodes (principalement Asellotes). Bull. Soc. Linn. Lyon 52 (10) : 319-357.

Hynes H.B.N., 1955. The reproductive cycle of some british freshwater Gammaridae. J. Anim. Ecol. (2) : 352-387.

Jazdzewski K., 1980. Range extensions of some gammaridean species in European inland water caused by human activity. Crustaceana, suppl. 6 : 84-107.

Kelleher B., Bergers P.J.M., Van den Brink F.W.B., Giller P.S., Van der Velde G. \& Bij de Vaate A., 1998. Effects of exotic amphipod invasions on fish diet in the Lower Rhine. Archiv für Hydrobiologie 143 (3) : 363-382.

Khalaf G. \& Tachet H., 1978. Un problème d'actualité : revue de travaux en matière d'utilisation des substrats artificiels pour l'échantillonnage des macroinvertébrés des eaux courantes. Bull. Ecol. 9 (1) : 29-38.

Nijssen H. \& Stock J.H., 1966. The Amphipod Gammarus tigrinus Sexton, 1939, introduced in the Netherlands
(Crustacea). Beaufortia 13 (160) : 197-206.

Pinkster S., Scheepmaker M., Platvoet D. \& Broodbakker N., 1992. Drastic changes in the amphipod fauna (Crustacea) of Dutch inland waters during the last 25 years. Bijdr. Dierk. 61 (4) : 193-204.

Pinkster S., Dieleman J. \& Platvoet D., 1980. The present position of Gammarus tigrinus Sexton, 1939, in the Netherlands, with the description of a newly discovered amphipod species, Crangonyx pseudogracilis Bousfield, 1958 (Crustacea, Amphipoda). Bull. Zool. Mus. Univ. Amsterdam 7 (4) : 35-45.

Roger M.C. \& Faessel B., 1989. Effets de l'échauffement artificiel de l'eau du Rhône sur le développement et la production des Gammaridae. Hydroécol. Appl. 1/2 : 53-83.

Thioulouse J., Chessel D., Dolédec S. \& Olivier J.M., 1997. ADE-4 : a multivariate and graphical display software. Stat. Comput. 7 : 75-83.

Vanden Bossche J.P., 2002. First record and fast spread of five new (19952000) alien species in the River Meuse in Belgium : Hypania invalida, Corbicula fluminea, Hemimysis anomala, Dikerogammarus villosus and Crangonyx pseudogracilis. Bull. inst. roy. sc. nat. Belgique 72 suppl. : 73-78.

Van den Brink F.W.B., Van der Velde G. \& Bij de Vaate A., 1991. Amphipod invasion on the Rhine. Nature 352 (6336) : 576.

Van Maren M.J., 1978. Distribution and ecology of Gammarus tigrinus Sexton, 1939 and some other amphipod Crustacea near Beaufort (North Carolina, USA). Bijdr. Dierk. 48 (1) : 45-56.

Wouters K., 2002. A review of neozan non-marine macro-crustaceans in Belgium. Bull. inst. roy. sc. nat. Belgique 72 suppl. : 83-85. 
